\title{
Article \\ Ultra-Low-Loss Mid-Infrared Plasmonic Waveguides Based on Multilayer Graphene Metamaterials
}

\author{
Chia-Chien Huang ${ }^{1,2, *}$, , Ruei-Jan Chang ${ }^{2}$ and Ching-Wen Cheng ${ }^{2}$ \\ 1 Institute of Nanoscience, National Chung Hsing University, Taichung 40227, Taiwan \\ 2 Department of Physics, National Chung Hsing University, Taichung 40227, Taiwan; \\ grunt230@yahoo.com.tw (R.-J.C.); mikasa210549@gmail.com (C.-W.C.) \\ * Correspondence: cch@phys.nchu.edu.tw
}

Citation: Huang, C.-C.; Chang, R.-J.; Cheng, C.-W. Ultra-Low-Loss Mid-Infrared Plasmonic Waveguides Based on Multilayer Graphene Metamaterials. Nanomaterials 2021, 11 2981. https://doi.org/10.3390/ 11112981

Academic Editor: Onofrio M. Maragò

Received: 13 October 2021

Accepted: 5 November 2021

Published: 6 November 2021

Publisher's Note: MDPI stays neutral with regard to jurisdictional claims in published maps and institutional affiliations.

Copyright: () 2021 by the authors. Licensee MDPI, Basel, Switzerland. This article is an open access article distributed under the terms and conditions of the Creative Commons Attribution (CC BY) license (https:// creativecommons.org/licenses/by/ $4.0 /)$.

\begin{abstract}
Manipulating optical signals in the mid-infrared (mid-IR) range is a highly desired task for applications in chemical sensing, thermal imaging, and subwavelength optical waveguiding. To guide highly confined mid-IR light in photonic chips, graphene-based plasmonics capable of breaking the optical diffraction limit offer a promising solution. However, the propagation lengths of these materials are, to date, limited to approximately $10 \mu \mathrm{m}$ at the working frequency $f=20 \mathrm{THz}$. In this study, we proposed a waveguide structure consisting of multilayer graphene metamaterials (MLGMTs). The MLGMTs support the fundamental volume plasmon polariton mode by coupling plasmon polaritons at individual graphene sheets over a silicon nano-rib structure. Benefiting from the high conductivity of the MLGMTs, the guided mode shows ultralow loss compared with that of conventional graphene-based plasmonic waveguides at comparable mode sizes. The proposed design demonstrated propagation lengths of approximately $20 \mu \mathrm{m}$ (four times the current limitations) at an extremely tight mode area of $10^{-6} A_{0}$, where $A_{0}$ is the diffraction-limited mode area. The dependence of modal characteristics on geometry and material parameters are investigated in detail to identify optimal device performance. Moreover, fabrication imperfections are also addressed to evaluate the robustness of the proposed structure. Moreover, the crosstalk between two adjacent present waveguides is also investigated to demonstrate the high mode confinement to realize high-density on-chip devices. The present design offers a potential waveguiding approach for building tunable and large-area photonic integrated circuits.
\end{abstract}

Keywords: graphene; multilayer; mid-infrared photonic; plasmonic waveguide; metamaterials; nano-optics; field enhancement

\section{Introduction}

Significant enhancements achieved in light-matter interactions, nonlinear optical effects, chemical and biological sensing sensitivities, and resolution in imaging and spectroscopy can further benefit from the localization of light waves in deep subwavelength sizes. To date, the most promising approach to achieve this goal is to excite surface plasmon polaritons (SPPs) [1] by coupling photons and free electrons at metal-dielectric interfaces. For visible and near-infrared (IR) wavelengths, noble metals have been used to build various nanoscale photonic circuits [2-5]. However, alternative metallic materials are necessary because of the weak confinement of SPP modes in conventional noble metals for mid-IR and terahertz (THz) bands [6,7]. Graphene [8-12] is an emerging 2D material with extraordinary electric, thermal, and optical properties that can be flexibly tuned by electrical gating or chemical doping. Graphene is considered a promising candidate for SPP waveguiding in the mid-IR $[6,7,9,11,12]$ and $\mathrm{THz}[9,12]$ ranges because of its nearly pure imaginary surface conductivity and extreme light confinement. The real and imaginary parts of the surface conductivity of graphene characterize the ohmic loss and magnitude of the wavevector, respectively. At the spectral bands of mid-IR and $\mathrm{THz}$, the real part of 
graphene's surface conductivity approaches zero, leading to a comparatively low ohmic loss. Because of their superior merits, many graphene-based optoelectronic and photonic devices, including polarizers [13-16], modulators [17-21], sensors [22-24], switches [25-28], and couplers [29], have been reported in recent years. To design functional optical devices well, detailed analyses of the mode properties of graphene plasmonic waveguides (GPWs) are of essential importance.

Many studies [30-39] have reported a variety of GPWs operating in the mid-IR range to improve SPP mode performance. For a plasmonic waveguide, several indices to evaluate the waveguiding performance of a device include the normalized mode area $\left(A_{m}\right)$; the propagation length $\left(L_{p}=\lambda /\left[4 \pi \operatorname{Im}\left(n_{e}\right)\right]\right)$, where $\lambda$ is the working wavelength in free space and $\operatorname{Im}\left(n_{e}\right)$ is the imaginary part of the effective refractive index; and the figure of merit $(F o M)$, which indicates the ratio between $L_{p}$ and mode size. For a dielectric loaded GPW, Xu et al. [31] theoretically investigated the dependence of the $L_{p}$ of fundamental graphene-based SPP (GSPP) modes on the geometry parameter, frequency $(f)$, Fermi energy $\left(E_{F}\right)$, and carrier mobility $(\mu)$ of graphene. At $f=20 \mathrm{THz}$, they obtained $L_{p}<3 \mu \mathrm{m}$ at $E_{F}=0.6 \mathrm{eV}$ and $\mu=1 \mathrm{~m}^{2} / \mathrm{V} \cdot \mathrm{s}$ (the same value is used for subsequent comparisons, unless stated otherwise). Note that the calculations of $L_{p}$ in Refs. [31-39] are unified by the same definition as mentioned above. Liu et al. [32] proposed a symmetrical long-range GSPP hybrid waveguide on a silica $\left(\mathrm{SiO}_{2}\right)$ buffer layer on a silicon $(\mathrm{Si})$ substrate showing an $L p$ of approximately $5 \mu \mathrm{m}$ and an extremely confined area, $A_{m}=8.0 \times 10^{-7}$, at $f=30 \mathrm{THz}$ and $E_{F}=0.8 \mathrm{eV}$. Considering a pattern-free suspended graphene sheet over a Si ridge substrate, Bahadori-Haghighi et al. [33] numerically demonstrated a low-loss GPW with $L_{p}=9 \mu \mathrm{m}$ at $f=30 \mathrm{THz}$ and $E_{F}=0.35 \mathrm{eV}$.

In addition to these GPWs [31-33], researchers have focused on another kind of GPW, called graphene-coated nanowire waveguide (GCNW)-related structures [34-39]. Gao et al. [34,35] showed that a single GCNW performs low propagation loss and is cutoff free for the fundamental mode. At $f=30 \mathrm{THz}$, a single GCNW with a radius of $R=100 \mathrm{~nm}$ and permittivity $\varepsilon_{N W}=2.1$ shows $L_{p}=4 \mu \mathrm{m}$ and $A_{m}=9.0 \times 10^{-4}$ at $E_{F}=0.6 \mathrm{eV}$ for the fundamental mode. To improve the weak confinement of a radically polarized mode in a single GCNW [34,35], Teng et al. [36] adopted a GCNW dimer to couple two GCNW modes. The mode properties were moderately improved to $L_{p}=4 \mu \mathrm{m}$ and $A_{m}=9.0 \times 10^{-5}$ at $E_{F}=0.6 \mathrm{eV}$ and $f=30 \mathrm{THz}$. To significantly enhance the mode localization, Liu et al. [37] reported a two-layer dielectric GCNW composed of a Si core surrounded by a $\mathrm{SiO}_{2}$ layer and an outermost graphene sheet, forming a conventional hybrid plasmonic waveguide (HPW) structure [40]. They obtained an $A_{m}$ of approximately $1.5 \times 10^{-5}$ and an $L_{p}$ of approximately $1.5 \mu \mathrm{m}$ at $E_{F}=0.5 \mathrm{eV}$ operating at a wavelength of $\lambda=7 \mu \mathrm{m}$ (about $f=42.85 \mathrm{THz}$ ). The cost for improving energy confinement is reflected in a moderately shorter $L_{p}$. Later, Liu et al. [38] extended their design by adding an extra graphene sheet between the $\mathrm{Si}$ and $\mathrm{SiO}_{2}$ layers to form a symmetric long-range coupling mode with an $L_{p}$ of $\sim 10 \mu \mathrm{m}$ and $A_{m}$ of $\sim 10^{-5}$ at $E_{F}=0.6 \mathrm{eV}$ and $f=30 \mathrm{THz}$. Last year, Teng et al. [39] adopted a GCNW-loaded Si nano-rib (GCNWLSNR) structure based on the coupling between the GCNW plasmon mode and the Si nano-rib to significantly shrink the mode size to $A_{m}=9.8 \times 10^{-7}$ while keeping $L_{p}$ at $\sim 9 \mu \mathrm{m}$ at $E_{F}=0.6 \mathrm{eV}$ and $f=20 \mathrm{THz}$. The experimental fabrication approach to a GCNW, by rolling a graphene ribbon, was presented in refs. $[41,42]$ to demonstrate practical feasibility.

In this study, we propose a high-performance GPW based on multilayer graphene metamaterials (MLGMTs) $[43,44]$ on a Si nano-rib waveguide partially covered by a lowindex porous $\mathrm{SiO}_{2}$ film. The fundamental volume plasmon polariton (VPP) modes, which are supported by MLGMT-coupling SPPs at individual graphene sheets [45], are coupled with a dielectric mode supported by a Si nano-rib waveguide. The resulting coupled mode performs not only deep subwavelength mode confinement but also ultra-low loss through introducing the high-conductivity MLGMTs compared with those of previously published results [32-39]. The proposed approach provides an additional degree of freedom, the number of graphene layers, to control the mode characteristics. Moreover, fabrication 
imperfections and spectral response are also addressed to evaluate the robustness and operating bandwidth of the proposed structure.

\section{Waveguide Structure and Methods}

A 3D schematic diagram of the GPW and its front view are shown in Figure 1a,b, respectively. The structure consists of a porous $\mathrm{SiO}_{2}[46,47]$ layer sandwiched by MLGMTs, which are formed by alternating graphene-dielectric layers, and a Si nano-rib waveguide deposited on a conventional $\mathrm{SiO}_{2}$ substrate. Here, the same $\mathrm{SiO}_{2}$ as the substrate is chosen as the dielectric layers in the MLGMTs. Following the HPW structure [40], the MLGMTs, porous $\mathrm{SiO}_{2}$, and $\mathrm{Si}$ nano-rib are considered the metal, low-index dielectric, and high-index dielectric layers, respectively. Therefore, the mode field of the present structure can be strongly squeezed in the nanoscale region between the Si nano-rib and the MLGMTs, thus achieving ultrasmall mode sizes.

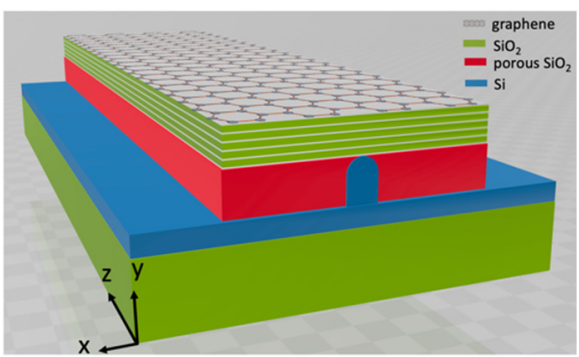

(a)

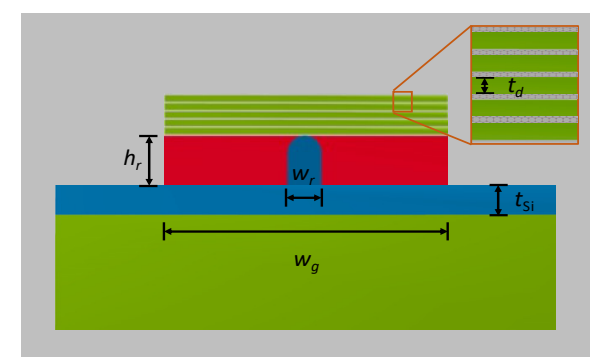

(b)

Figure 1. Schematic of the proposed waveguide structure-(a) 3D structure and (b) cross section of (a) with a zoomed-in view of the MLGMTs.

The fabrication steps are schematically shown in Figure 2 and are described as follows:

(1) Si and PR depositions

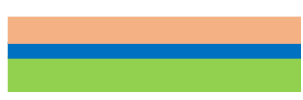

(6) Mask, PR exposure, and development

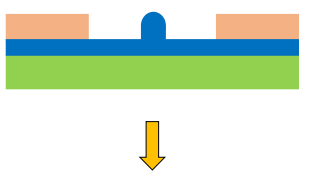

(7) Porous $\mathrm{SiO}_{2}$ deposition, PR lift out, and CMP
(2) Mask, PR exposure, and development

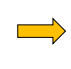

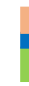

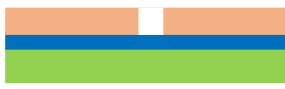

(5) PR coating and CMP

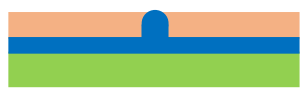

(8) Multilayer graphene-SiO stacking by layer-transfer
(3) Si deposition and PR lift off

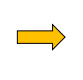

(4) Rounding top by e-beam

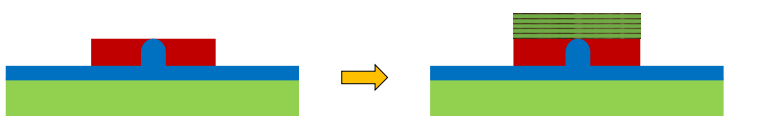

Figure 2. Schematic of the fabrication process for the proposed waveguide structure.

(1) Si and positive photoresist (PR) films with thickness $h_{r}(\mathrm{~nm})$ are deposited on a conventional $\mathrm{SiO}_{2}$ substrate; (2) a mask is applied and followed by PR exposure and PR development to define a rectangular groove; (3) a Si layer is deposited and followed by the lift off of the PR to form a Si nano-rib waveguide; (4) the top of the Si nano-rib is rounded using e-beam lithography by carefully controlling the exposure time and scanning speed; (5) a PR film is coated on the surface and is used to flatten the PR film using chemical mechanical polishing (CMP); (6) a mask is applied and followed by PR exposure and 
PR development to lift out the PR film and define the porous $\mathrm{SiO}_{2}$ region; (7) a porous $\mathrm{SiO}_{2}$ film is evaporated on the surface using the oblique deposition technique [46,47], the PR film is lifted off, and then CMP is used to flatten the porous $\mathrm{SiO}_{2}$ layer; finally, (8) forming multilayer graphene- $\mathrm{SiO}_{2}$ stacking by a chemical vapor deposition (CVD), a layer transfer method [48-50], or transfer-free, solution-phase deposition method [51,52]. Considering the high-technique step to form the MLGMTs, we describe the main fabrication processes as follows. The former approaches [48-50] include depositing graphene on a copper foil, transferring it to the substrate using the PMMA transfer technique, etching the copper foil using ammonium persulfate, doping the graphene by soaking the sample in a suitable solution, depositing the target dielectric layer by the atomic layer deposition being able to carefully control its thickness, and finally repeating the processes to achieve the desired number of layers. Note that the number of graphene layers in [50] can reach 11 layers. For the latter approach [51,52], the fabrication processes include the use of graphene-oxide (GO) in solution, deposition directly on a dielectric layer without requiring a transfer process, repetition to achieve the desired number of GO layers, and finally laser photoreduction from GO to graphene with removing the oxygen-functional groups, in which the bandgap of the graphene can be tuned by varying the lase power. Note that the number of graphene layers in [52] can reach 20 layers. Compared to the CVD transfer method [48-50], the advantages of the solution-phase method [51,52] are transfer-free, with quality independent of the number of layers, and controllable for the optical responses by tunning the bandgap of graphene. Consequently, the fabrication of the multilayer graphene metamaterials may be not that challenging and have complex techniques required and is moderately achievable using modern fabrication techniques, making the proposed waveguide structure practically realizable. Note that the conventional electrical gating on graphene layers uses a single voltage to a top contact $[53,54]$, thus resulting in an inhomogeneous chemical potential of the graphene layers varying from layer to layer due to interlayer screening [55] in a multilayer graphene structure. The non-uniformity of chemical potential is more significant as the number of graphene layers increases. As a result, a potential scheme [49] can be adopted to achieve the required chemical potential in the proposed MLGHPW. This approach controls the Fermi energy levels of individual graphene layers by different gate voltages, making the carrier concentrations alter together in all layers.

In theory, the MLGMTs can be considered a coupled system with interacting multiple graphene sheets supporting multiple nondegenerate plasmon modes (called VPP modes). The VPP modes show hyperbolic isofrequency contours [45] and a large density of electromagnetic states, resulting in high- $k$ guided modes. Among the VPP modes, the fundamental VPP mode $\left(\mathrm{VPP}_{0}\right)$ shows the lowest loss, although it possesses a comparably larger mode size. By coupling the $\mathrm{VPP}_{0}$ with the dielectric mode of the Si nano-rib waveguide, a hybrid mode not only preserves the low-loss property of the $\mathrm{VPP}_{0}$, but also significantly benefits from the high mode confinement of the Si nano-rib. In the present structure, the geometry parameters are as follows: $w_{g}$ and $t_{d}$ are the width and thickness, respectively, of the dielectric $\left(\mathrm{SiO}_{2}\right)$ layer; $N$ is the number of MLGMT layers between graphene sheets ( $N+1$ layers); and $w_{r}, h_{r}$, and $t_{S i}$ are the width, height, and bottom thickness of the Si nano-rib waveguide, respectively. Here, we set the radius of curvature to $r=w_{r} / 2$. The relative permittivities of $\mathrm{Si}$, conventional $\mathrm{SiO}_{2}$, and porous $\mathrm{SiO}_{2}$ at $f=20 \mathrm{THz}$ are $\varepsilon_{S i}=12.25$ [56], $\varepsilon_{\mathrm{SiO}_{2}}=2.25$ [56], and $\varepsilon_{p-S i O_{2}}=1.10$ [46], respectively. Here, graphene is modeled as an infinitely thin sheet with a surface current density of $J=\sigma E$ in-plane, where $E$ is the electric field vector and $\sigma$ is the total surface conductivity of graphene with $\sigma=\sigma_{\text {intra }}+\sigma_{\text {inter }}$, including intraband $\left(\sigma_{\text {intra }}\right)$ and interband $\left(\sigma_{\text {inter }}\right)$ contributions, which can be calculated using the local random phase approximation [57]:

$$
\sigma_{\text {intra }}\left(\omega, E_{F}, \tau, T\right)=\frac{-j 2 e^{2} k_{B} T}{\pi \hbar^{2}\left(\omega-j \tau^{-1}\right)} \ln \left[2 \cosh \left(\frac{E_{F}}{2 k_{B} T}\right)\right]
$$


and

$$
\sigma_{\text {inter }}\left(\omega, E_{F}, \tau, T\right)=\frac{e^{2}}{4 \hbar} \ln \left[\begin{array}{c}
\frac{1}{2}+\frac{1}{\pi} \tan ^{-1}\left(\frac{\hbar \omega-2 E_{F}}{2 k_{B} T}\right)+ \\
\frac{j}{2 \pi} \ln \left(\frac{\left(\hbar \omega+2 E_{F}\right)^{2}}{\left(\hbar \omega-2 E_{F}\right)^{2}+\left(2 k_{B} T\right)^{2}}\right)
\end{array}\right],
$$

where $E_{F}$ is the Fermi energy, $\tau=\mu E_{F} / e V_{F}^{2}$ is the carrier relaxation lifetime, $T$ is the temperature, $k_{B}$ is the Boltzmann constant, $\hbar$ is the reduced Planck constant, $e$ is the electron charge, $\mu$ is the carrier mobility in graphene, and $V_{F}=10^{6} \mathrm{~m} / \mathrm{s}$ is the Fermi velocity of electrons. Here, we consider a carrier mobility, $\mu=1 \mathrm{~m}^{2} / \mathrm{V} \cdot \mathrm{s}$, at $T=300 \mathrm{~K}$, which is also used to study the performance of GPWs [34-39]. To evaluate the waveguiding performance of plasmonic waveguides, the propagation length $\left(L_{p}\right), F o M\left(L_{p} / 2 \sqrt{A_{m} / \pi}[40]\right)$, and normalized mode area $\left(A_{m}=A_{e} / A_{0}\right)$ are used, where $A_{0}=\lambda^{2} / 4$ ( $\lambda$ is the working wavelength) is the diffraction-limited mode area and $A_{e}$ is the effective mode area given by

$$
A_{e}=\frac{W_{m}}{W(r)_{\max }}=\frac{1}{W(r)_{\max }} \int_{-\infty}^{\infty} \int_{-\infty}^{\infty} W(r) d A .
$$

Equation (3) includes the ratio of the total mode energy, $W_{m}$, and the peak of the energy density, $W(r)$, which is given by:

$$
W(r)=\frac{1}{2}\left\{\operatorname{Re}\left[\frac{d \varepsilon(r) \omega}{d \omega}\right]|\mathbf{E}(r)|^{2}+\mu_{0}|\mathbf{H}(r)|^{2}\right\},
$$

where $\omega$ is the angular frequency, $\varepsilon(r)$ is the profile of relative permittivity, $\mu_{0}$ is the permeability in a vacuum, and $|\mathbf{E}|$ and $|\mathbf{H}|$ are the intensities of the electric and magnetic fields, respectively. The numerical results are calculated using the COMSOL Multiphysics software based on the rigorous finite element method.

\section{Results and Discussion}

\subsection{Waveguiding Performance Dependence on the Number of MLGMT Graphene Layers}

Figure 3 shows the mode properties of the present structure versus $N$ for several values of $t_{S i}$ at the following parameters: $w_{g}=200 \mathrm{~nm}, t_{d}=5 \mathrm{~nm}, w_{r}=10 \mathrm{~nm}, h_{r}=30 \mathrm{~nm}$, $E_{F}=0.6 \mathrm{eV}$, and $f=20 \mathrm{THz}$. We observe that the real part of $n_{e}, \operatorname{Re}\left(n_{e}\right)$ sharply decreases from approximately 20 to 5 as $N$ increases (see Figure 3a), but $A_{m}$ moderately increases from $5.34 \times 10^{-7}$ to $1.93 \times 10^{-6}$ for $t_{S i}=5 \mathrm{~nm}$ (see Figure 3b). Note that the slopes of $\operatorname{Re}\left(n_{e}\right)$ and $A_{m}$ versus $N$ become smaller when $N$ is greater than five, meaning that $\operatorname{Re}\left(n_{e}\right)$ and $A_{m}$ are slightly influenced by larger $N$ values. In contrast, $L_{p}$ linearly increases from 5.1 to $27.9 \mu \mathrm{m}$ for $t_{S i}=5 \mathrm{~nm}$ as $N$ increases from 0 to 10 (see Figure 3c). Further increasing $L_{p}$ is only limited by the ability to fabricate an increasing number of $N$. The FoM increases from 6214 at $N=0$ to 17,830 at $N=10$ for $t_{S i}=5 \mathrm{~nm}$ (see Figure $3 \mathrm{~d}$ ).

The obtained results show that the proposed design can achieve both an ultralong $L_{p}$ (up to $\sim 28 \mu \mathrm{m}$ ) and an extremely small $A_{m}$ (on the order of $10^{-6}$ ). Comparing these values with $A_{m}=9.8 \times 10^{-7}$ and $L_{p}=3.5 \mu \mathrm{m}$ of the GCNWLSNR [39], the present structure improves $L_{p}$ by more than three times while maintaining a comparable order of magnitude of $A_{m}$. For observing the mode profiles, Figure $4 \mathrm{a}-\mathrm{c}$ depict the $|\mathbf{E}|$ values near the nanoscale region between the MLGMTs and Si nano-rib for $N=1,5$, and 10 at $t_{S i}=5 \mathrm{~nm}$. Clearly, increasing $N$ results in a stronger peak of $|\mathrm{E}|$ but looser mode confinement, leading to the moderate increase in $A_{m}$ (see Figure 3b). 


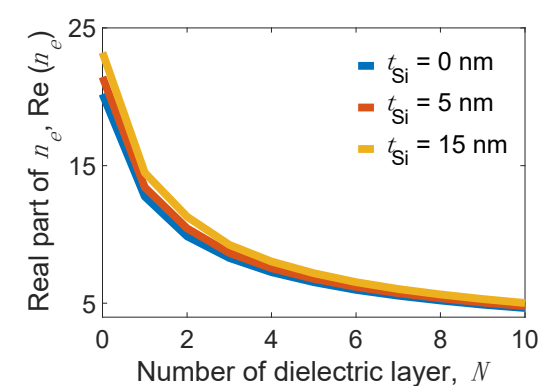

(a)

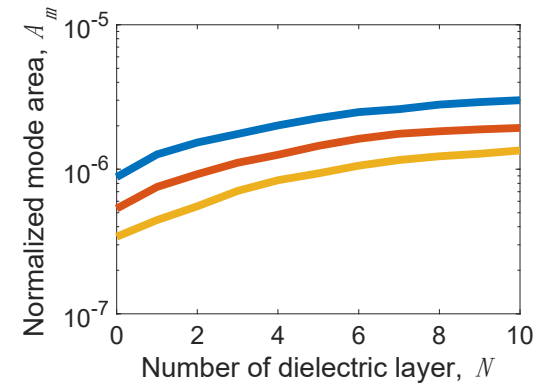

(c)

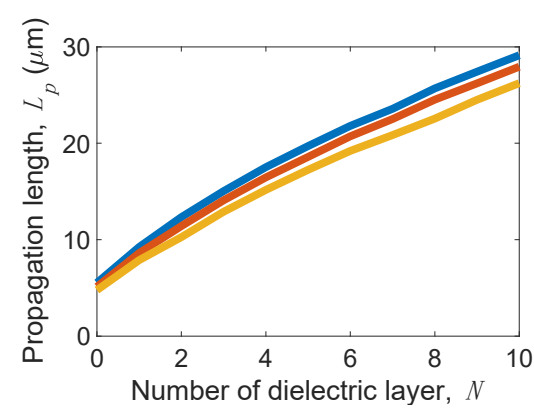

(b)

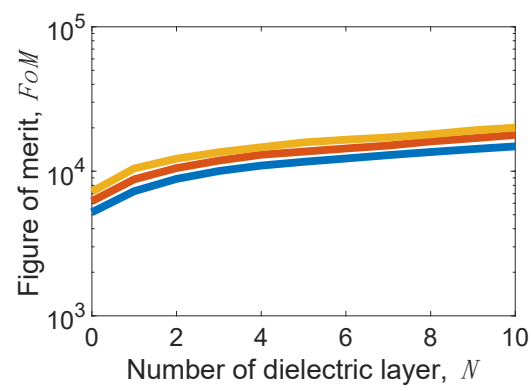

(d)

Figure 3. (a) Real part of effective index $n_{e}, \operatorname{Re}\left(n_{e}\right)$, (b) normalized mode area, $A_{m}$, (c) propagation length, $L_{p}$, and (d) figure of merit, FoM versus the number of dielectric layer, $N$ for the present structure operating at $f=20 \mathrm{THz}$ for different $t_{S i}$ values at parameters of $w_{g}=200 \mathrm{~nm}, t_{d}=5 \mathrm{~nm}$, $w_{r}=10 \mathrm{~nm}, h_{r}=30 \mathrm{~nm}$, and $E_{F}=0.6 \mathrm{eV}$.

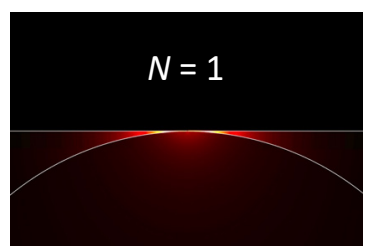

(a)

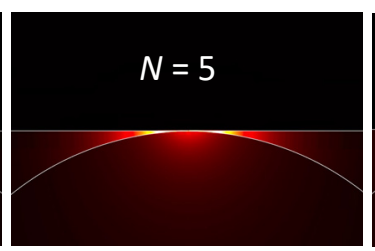

(b)

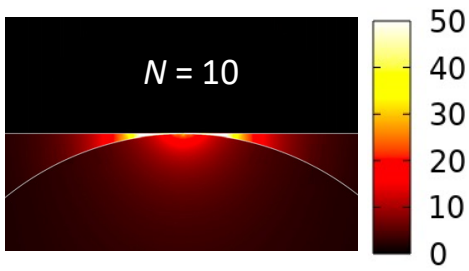

(c)

Figure 4. Mode fields of $|\mathbf{E}|$ for $N=(\mathbf{a}) 1,(\mathbf{b}) 5$, and (c) 10 at the same parameters as in Figure 3.

Figure 5a,b also show 1D field plots along the horizontal dashed line $H$ (inset of Figure 5a) and vertical dashed line $V$ (inset of Figure $5 \mathrm{~b}$ ), respectively, to more clearly observe the relative mode profiles for different $N$ values. The ultrasmall $A_{m}=10^{-6}$ of the present design shows full widths at half maximums of approximately 1 and $0.1 \mathrm{~nm}$ along the $x$ and $y$ directions, respectively. The $L_{p}$ can be attributed to the mode profile being significantly shifted from the region of MLGMTs to the Si nano-rib as $N$ increases (see Figure $5 b$ ). Note that the conditions $E_{F}=0.6 \mathrm{eV}, N=10$, and $f=20 \mathrm{THz}$ are used in subsequent analyses unless stated otherwise.

To clearly elucidate how the dielectric $\mathrm{Si}$ nano-rib changes the $\mathrm{VPP}_{0}$ mode, we analyze the properties of the $\mathrm{VPP}_{0}$ mode supported by the proposed structure without the nano-rib. Figure 6 shows the mode properties of the present structure with and without the nano-rib versus $N$ at the same parameters as used in Figure 3 with $t_{S i}=5 \mathrm{~nm}$. 


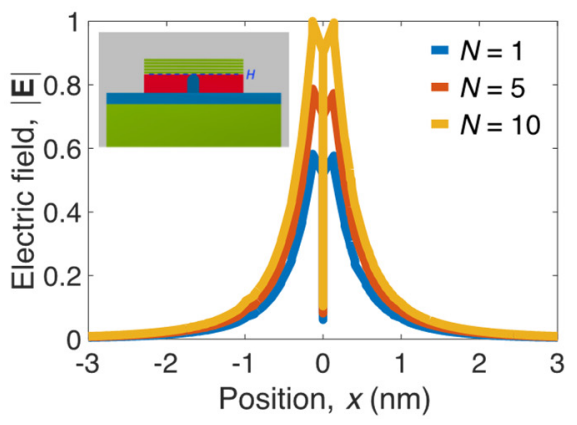

(a)

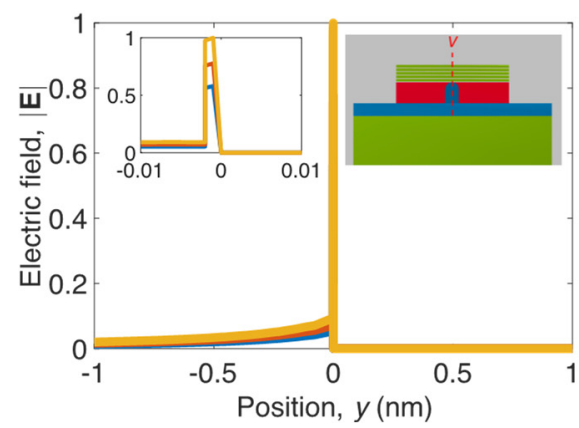

(b)

Figure 5. Electric fields, $|\mathbf{E}|$ along the (a) horizontal dashed line $H$ and (b) vertical dashed line $V$ shown in the corresponding insets for $N=1,5$, and 10 .

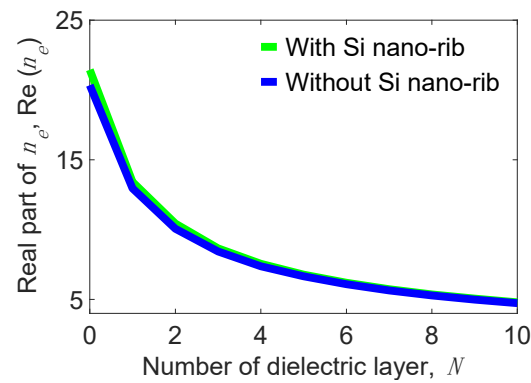

(a)

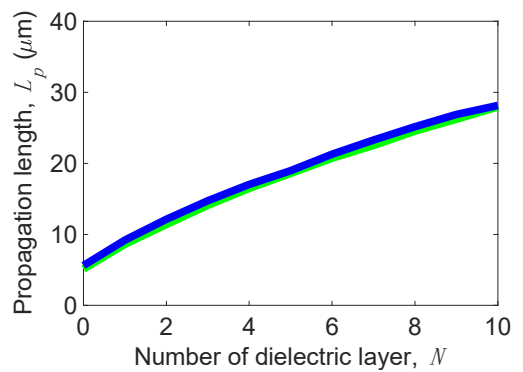

(c)

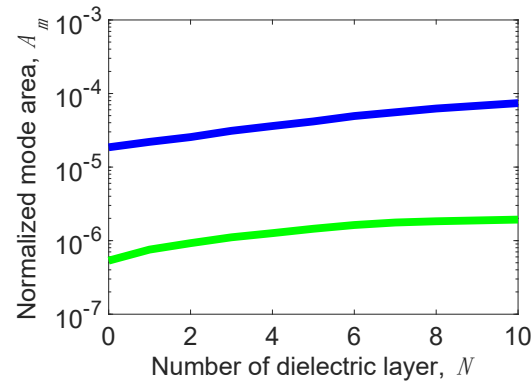

(b)

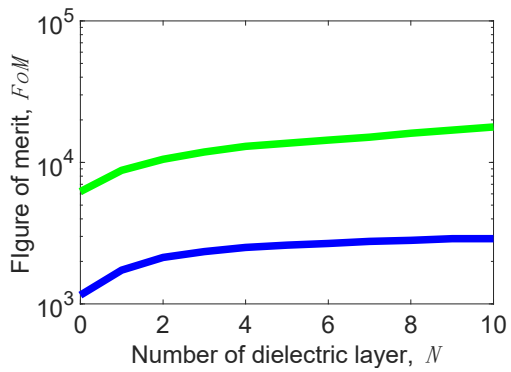

(d)

Figure 6. (a) Real part of effective index $n_{e}, \operatorname{Re}\left(n_{e}\right)$, (b) normalized mode area, $A_{m}$, (c) propagation length, $L_{p}$, and (d) figure of merit, FoM versus the number of dielectric layer $N$ for the present structure with and without the Si nano-rib.

We observe that $\operatorname{Re}\left(n_{e}\right)$ and $L_{p}$ of the two structures are really close, but $A_{m}$ of the structure without the nano-rib is one to two orders of magnitude larger than that of the present structure as shown in Figure 6b, making the FoM of the structure without the nano-rib about one order of magnitude smaller. The above result reveals that the nanorib can make the field distribution of the $\mathrm{VPP}_{0}$ mode significantly concentrated around the nanoscale region between the nano-rib and the MLGMTs. For observing the effect, Figure $7 \mathrm{a}, \mathrm{b}$ show the $|\mathrm{E}|$ distributions of the present structure with and without the nano-rib, respectively, along with the zoomed-in view (see inset of Figure $7 \mathrm{~b}$ ) of the | E | distribution around the nano-rib. Without the nano-rib, the field profile spreads stronger out of the MLGMTs than that of the present design. In addition, the field of the present structure is significantly enhanced and focused mainly around the nano-rib, effectively shrinking its $A_{m}$. 


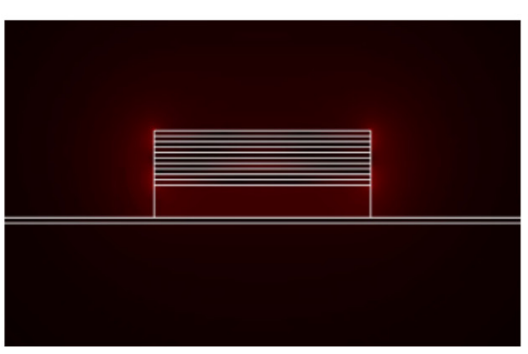

(a)

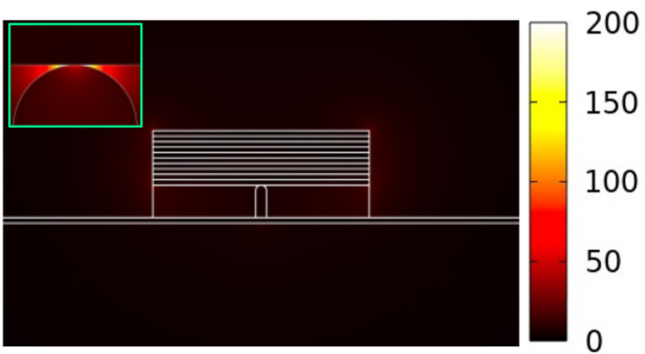

(b)

Figure 7. Mode fields of $|\mathbf{E}|$ for the present waveguide structure (a) without and (b) with the Si nano-rib.

\subsection{Mode Characteristic Dependence on Geometric Parameters}

To fully assess the waveguiding performance of the proposed structure, we analyze the geometrical dependence of the mode properties. Figure 8 shows the mode properties versus $w_{r}$ for several $h_{r}$ values at $w_{g}=200 \mathrm{~nm}, t_{S i}=5 \mathrm{~nm}$, and $t_{d}=5 \mathrm{~nm}$. We observe that $\operatorname{Re}\left(n_{e}\right)$ slightly depends on $w_{r}$ and $h_{r}$ (see Figure 8a) but $A_{m}$ shows a substantial dependence on $w_{r}$ and $h_{r}$ (see Figure $8 \mathrm{~b}$ ). For example, $A_{m}$ varies from $1.6 \times 10^{-6}$ to $6.0 \times 10^{-6}$, while $w_{r}$ changes from 5 to $35 \mathrm{~nm}$ at $h_{r}=30 \mathrm{~nm}$. This effect can be attributed to a larger $w_{r}$, obviously leading to a looser mode confinement. On the other hand, larger $h_{r}$ attains tighter $A_{m}$ due to the increase in the area of a low-index region, making the mode field more concentrated towards the region between the MLGMTs and the Si nano-rib. However, $L_{p}$ significantly increases as $h_{r}$ increases, although $A_{m}$ shrinks. This is because the mode field shifts toward the Si nano-rib side as $h_{r}$ increases. Interestingly, $L_{p}$ slightly increases for the smaller $h_{r}=10 \mathrm{~nm}$ but moderately decreases for the larger $h_{r}>30 \mathrm{~nm}$ as $w_{r}$ increases (see Figure 8c). For example, $L_{p}$ varies from 29.2 (25.8) at $w_{r}=5 \mathrm{~nm}$ to 26.8 (26.2) $\mu \mathrm{m}$ at $w_{r}=35 \mathrm{~nm}$ for $h_{r}=50(10) \mathrm{nm}$. Note that smaller $w_{r}$ or larger $h_{r}(>20 \mathrm{~nm}$ here) can effectively improve the FoM of the proposed waveguide structure (see Figure 8d).

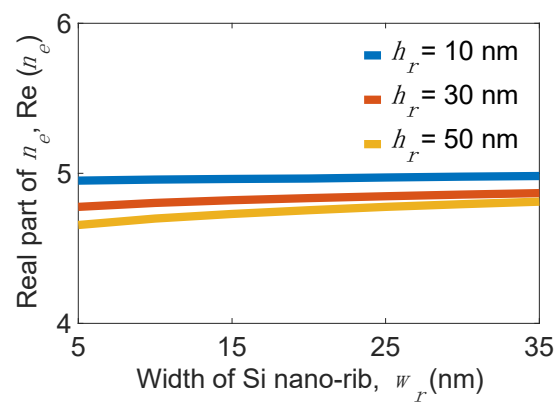

(a)

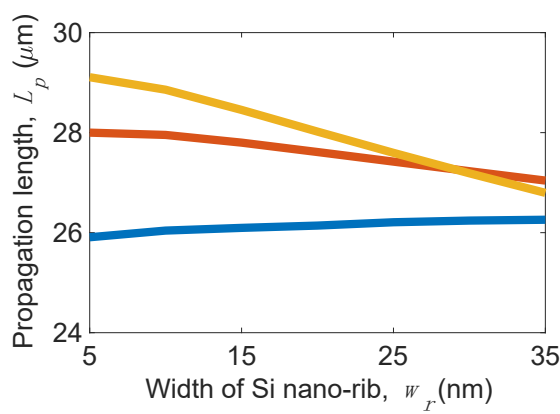

(c)

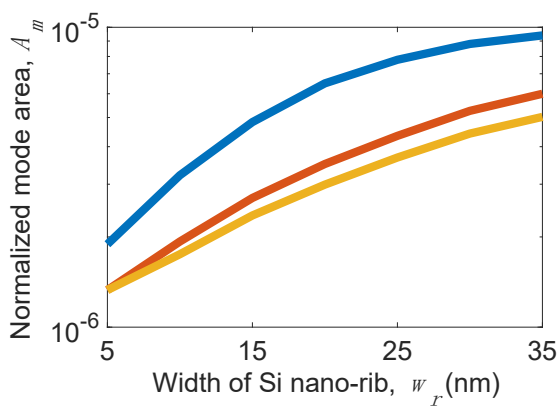

(b)

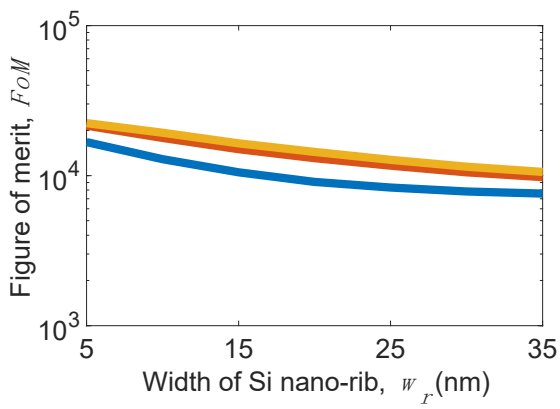

(d)

Figure 8. (a) Real part of effective index $n_{e}, \operatorname{Re}\left(n_{e}\right)$, (b) normalized mode area, $A_{m}$, (c) propagation length, $L_{p}$, and (d) figure of merit, FoM of the present structure versus the width of Si nano-rib, $w_{r}$ for different height of Si nano-rib, $h_{r}$ values at the width of MLGMTs, $w_{g}=200 \mathrm{~nm}$, thickness of the bottom Si layer, $t_{S i}=5 \mathrm{~nm}$, and thickness of dielectric layers of MLGMTs, $t_{d}=5 \mathrm{~nm}$. 
Next, we consider the effects of $w_{g}$ and $t_{d}$ of the MLGMTs on mode properties. At $w_{r}=10 \mathrm{~nm}, h_{r}=30 \mathrm{~nm}$, and $t_{S i}=5 \mathrm{~nm}$, Figure 9 shows the mode properties versus $w_{g}$ for several $t_{d}$ values. We observe that $\operatorname{Re}\left(n_{e}\right), A_{m}$, and $L_{p}$ moderately depend on $w_{g}$ and $t_{d}$. We also observe that $A_{m}$ and $L_{p}$ decrease as $w_{g}$ decreases. Differing from increasing $h_{r}$, which leads to a smaller $A_{m}$ (see Figure $8 \mathbf{b}$ ), increasing $w_{g}$ increases $A_{m}$, although they all increase the low-index region. This is because increasing $w_{g}$ also increases the width of the MLGMTs, leading to a looser mode field. The compensation between $A_{m}$ and $L_{p}$ makes the FoM almost constant for different values of $w_{g}$. As for the $t_{d}$, the smaller value leads to stronger coupling between SPP modes at individual graphene sheets. Therefore, smaller $t_{d}$ concurrently achieves lower loss and smaller mode size, breaking the trade-off between $A_{m}$ and $L_{p}$ to effectively improve the waveguiding performance of the proposed structure.

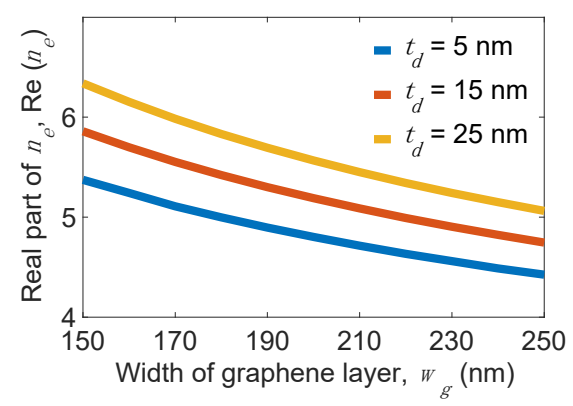

(a)

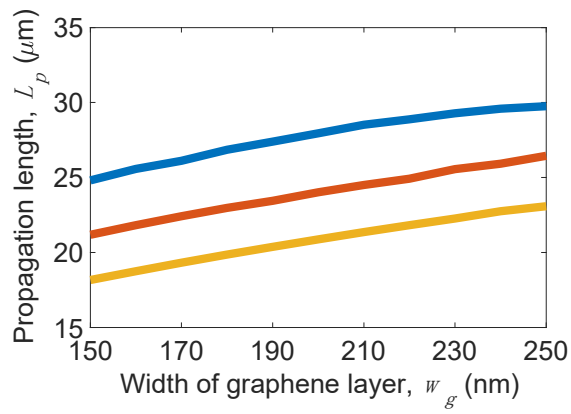

(c)

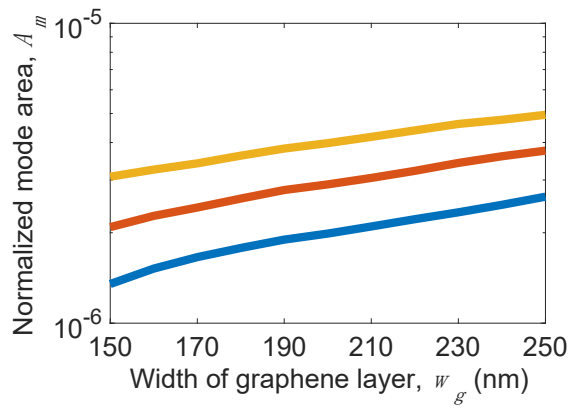

(b)

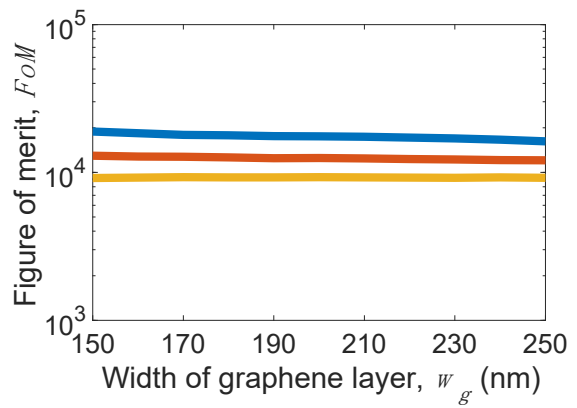

(d)

Figure 9. (a) Real part of effective index $n_{e}, \operatorname{Re}\left(n_{e}\right)$, (b) normalized mode area, $A_{m}$, (c) propagation length, $L_{p}$, and (d) figure of merit, FoM, of the present structure versus $w_{g}$ for different $t_{d}$ values at $w_{r}=10 \mathrm{~nm}, h_{r}=30 \mathrm{~nm}$, and $t_{S i}=5 \mathrm{~nm}$.

\subsection{Fabrication Tolerance, Material Parameters of Graphene, and Spectral Response}

In experiments, fabrication imperfections lead to a reduction in waveguiding performance. Among the geometric parameters, the strictest part of the structure that should be precisely fabricated is the dimension of the $\mathrm{Si}$ nano-rib. Figure 10a,b show the dependence of $A_{m}$ and $L_{p}$ on the relative fabrication error, $\Delta x / w_{r}$, at $h_{r}=30 \mathrm{~nm}, t_{S i}=5 \mathrm{~nm}, t_{d}=5 \mathrm{~nm}$, and $w_{g}=200 \mathrm{~nm}$, where $\Delta x$ is the fabrication error. Evidently, $A_{m}$ and $L_{p}$ are almost invariant in the range between $\Delta x / w_{r}=0$ and 0.5. Similarly, the dependence of $A_{m}$ and $L_{p}$ on the relative fabrication error $\Delta r / r$, where $\Delta r$ is the fabrication error, are shown in Figure 10c, d, respectively. At $r=10 \mathrm{~nm}, A_{m}$ moderately varies from $4.2 \times 10^{-6}$ to $8.5 \times 10^{-6}$ while $\Delta r / r$ deviates from 0 to 0.5 , but $L_{p}$ remains constant, although $\Delta r / r=0.5$. The analyzed results verify that the proposed structure possesses high fabrication tolerance on mode properties. 


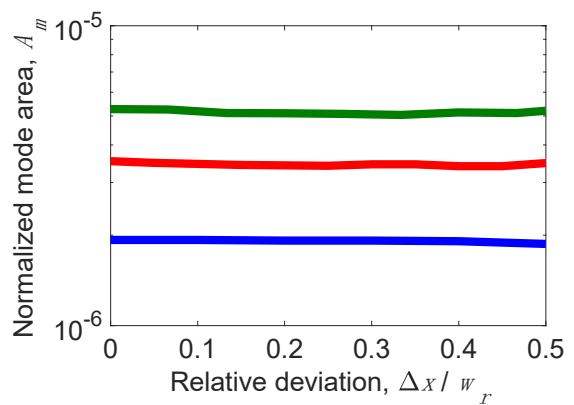

(a)

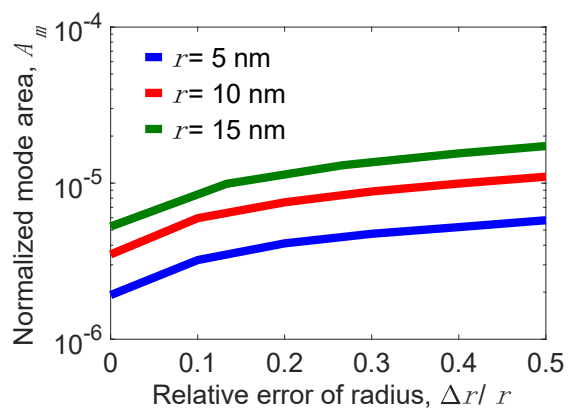

(c)

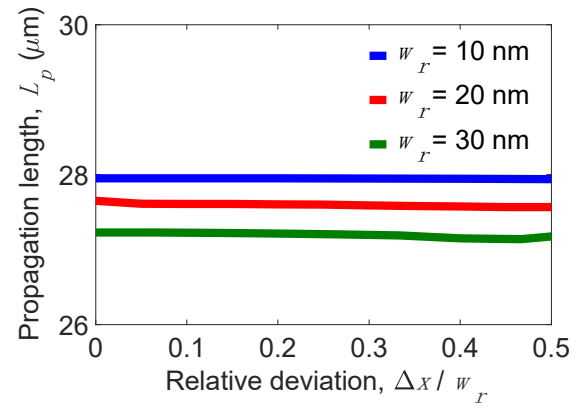

(b)

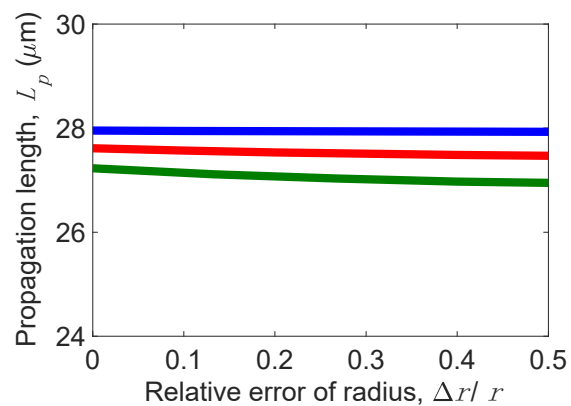

(d)

Figure 10. Dynamics of (a) normalized mode area, $A_{m}$, and (b) propagation length, $L_{p}$, versus the relative fabrication error of the width of the Si nano-rib $\left(\Delta x / w_{r}\right)$ for different $w_{r}$ values; (c) $A_{m}$ and (d) $L_{p}$ versus the relative fabrication error of the radius of the curvature of the Si nano-rib $(\Delta r / r)$ for different $r$ values when $h_{r}=30 \mathrm{~nm}, t_{S i}=5 \mathrm{~nm}, t_{d}=5 \mathrm{~nm}$, and $w_{g}=200 \mathrm{~nm}$.

Considering a high doping level of graphene that leads to reducing the carrier mobility $(\mu)$, we investigated the mode properties versus $\mu$ for several $E_{F}{ }^{\prime}$, as shown in Figure 11. We observe that $\operatorname{Re}\left(n_{e}\right)$ and $A_{m}$ are nearly independent on $\mu$; however, $L_{p}$ linearly reduces as $\mu$ decreases due to increasing the ohmic loss significantly for a higher doping level of $E_{F}>0.5 \mathrm{eV}$. At $E_{F}=0.4 \mathrm{eV}, L_{p}$ varies from 10.7 to $7.4 \mu \mathrm{m}$ at $\mu=1$ and $0.6\left(\mathrm{~m}^{2} / \mathrm{V} \cdot \mathrm{s}\right)$, respectively. On the other hand, a lower $E_{F}$ attains a moderately higher $\operatorname{Re}\left(n_{e}\right)$ and slightly smaller $A_{m}$ showing a tighter mode confinement, but significantly leads to a shorter $L_{p}$ (see Figure 11c). For example, $L_{p}$ varies from 19.5 to $10.8 \mu \mathrm{m}$ at $E_{F}=0.5$ and $0.4 \mathrm{eV}$, respectively, for the condition $\mu=1\left(\mathrm{~m}^{2} / \mathrm{V} \cdot \mathrm{s}\right)$. The results reveal that a lower $E_{F}$ or $\mu$ leads to a reduction in $L_{p}$ for general GPWs, mainly due to the higher ohmic losses.

To fully study the tunability of graphene within a bandwidth range, we address the spectral response of mode properties. At $w_{g}=200 \mathrm{~nm}, w_{r}=10 \mathrm{~nm}, h_{r}=30 \mathrm{~nm}$, $t_{S i}=5 \mathrm{~nm}$, and $t_{d}=5 \mathrm{~nm}$, the results for several values of $E_{F}$ are shown in Figure 12. As the working frequency, $f$, increases from 10 to $30 \mathrm{THz}, \operatorname{Re}\left(n_{e}\right)$ and $A_{m}$ moderately increase, but $L_{p}$ significantly decreases. Considering the effect of $E_{F}, \operatorname{Re}\left(n_{e}\right)$ increases, but $A_{m}$ and $L_{p}$ decrease because of the enhanced mode localization as $E_{F}$ decreases. Note that $A_{m}$ is slightly influenced while $L_{p}$ is significantly influenced by varying $E_{F}$. At $f=10(30) \mathrm{THz}$, our design achieves unprecedented long $L_{p}$ values of 12.1 (4.8) and 38.9 (19.4) $\mu \mathrm{m}$ while maintaining ultrasmall $A_{m}$ values of $6.8 \times 10^{-7}\left(1.8 \times 10^{-6}\right)$ and $7.8 \times 10^{-7}\left(2.6 \times 10^{-6}\right)$ for $E_{F}=0.4$ and $0.6 \mathrm{eV}$, respectively. Exceptionally, the obtained $F o M$ values are higher than $10^{4}\left(3.0 \times 10^{3}\right)$ within $f=10-30 \mathrm{THz}$, even while operating at $E_{F}=0.6(0.3) \mathrm{eV}$. 


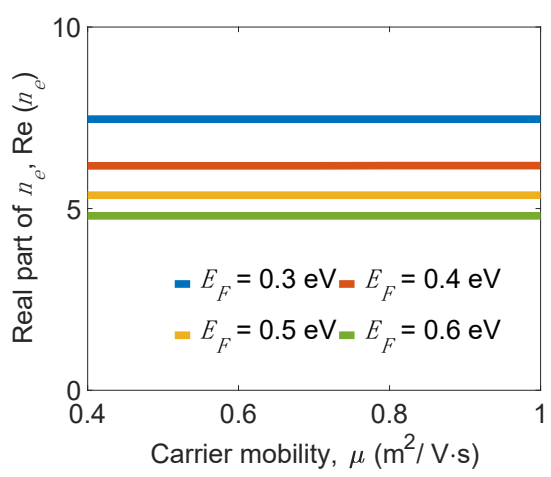

(a)

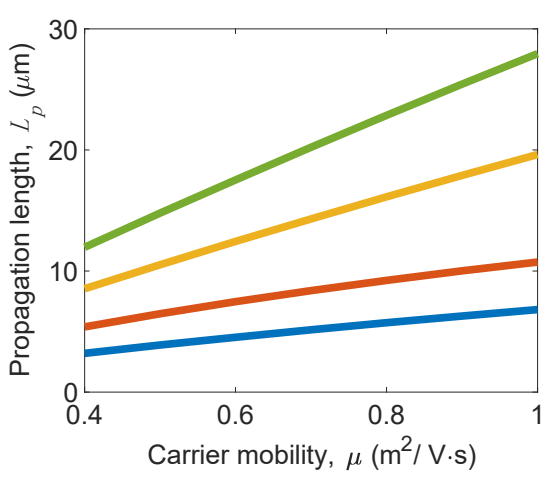

(c)

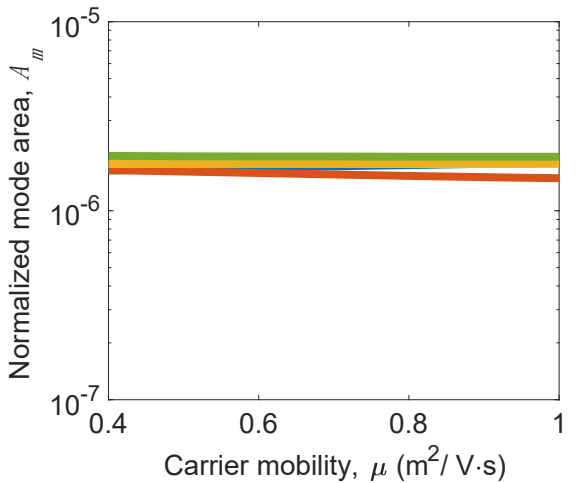

(b)

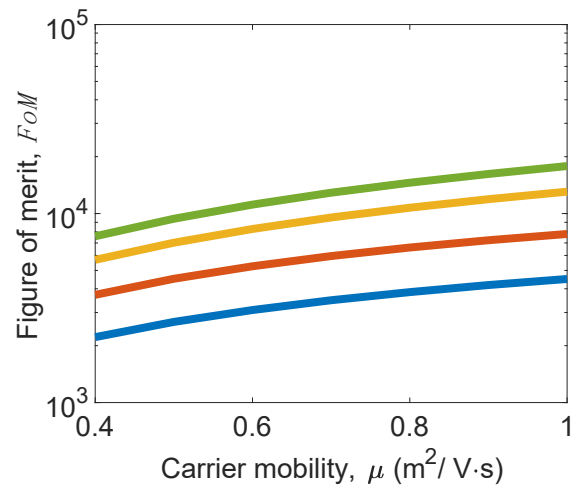

(d)

Figure 11. Plots of (a) real part of effective index $n_{e}, \operatorname{Re}\left(n_{e}\right),(\mathbf{b})$ normalized mode area, $A_{m},(\mathbf{c})$ propagation length, $L_{p}$, and (d) figure of merit, FoM, of the present structure versus carrier mobility, $\mu$, for several $E_{F}$ 's at $w_{g}=200 \mathrm{~nm}, w_{r}=10 \mathrm{~nm}, h_{r}=30 \mathrm{~nm}, t_{S i}=5 \mathrm{~nm}, t_{d}=5 \mathrm{~nm}$, and $f=20 \mathrm{THz}$.

The conventional electrical gating on graphene layers uses a single voltage to a top contact [50,51], thus resulting in an inhomogeneous chemical potential of the graphene layers varying from layer to layer due to interlayer screening [52] in a multilayer graphene structure. The non-uniformity of chemical potential is more significant as the number of graphene layers increases. As a result, a potential scheme [49] can be adopted to achieve the required chemical potential in the proposed MLGHPW. This approach controls the Fermi energy levels of individual graphene layers by different gate voltages, making the carrier concentrations alter together in all layers.

\subsection{Waveguide Crosstalk}

In addition to the size of $A_{m}$, the crosstalk of the modes in adjacent waveguides complements to describe the degree of mode confinement and examines the feasibility for high integration of photonic integrated circuits. Figure 13 shows a coupled waveguide consisting of two parallel waveguides with a center-to-center separation $s$. 


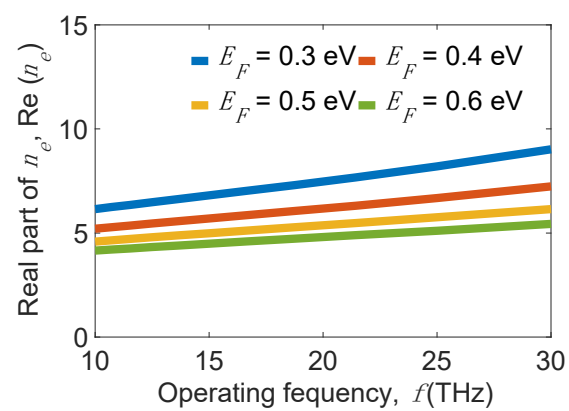

(a)

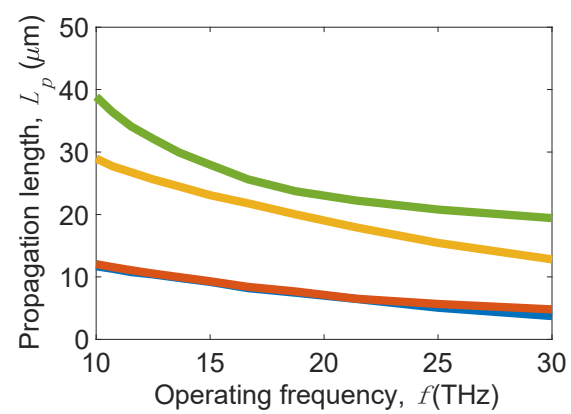

(c)

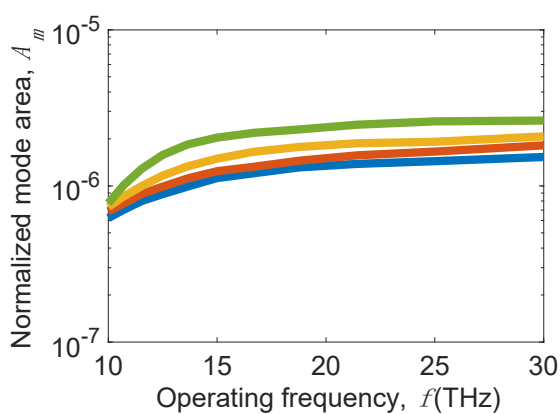

(b)

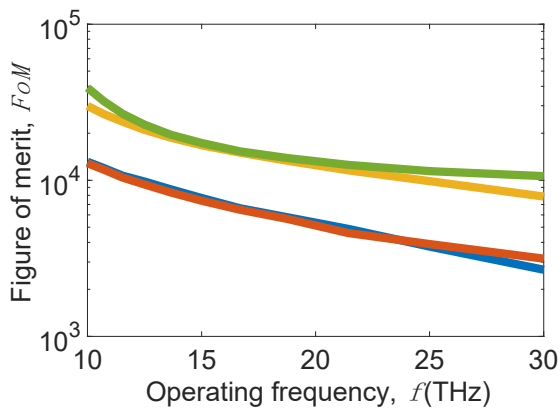

(d)

Figure 12. Plots of (a) real part of effective index $n_{e}, \operatorname{Re}\left(n_{e}\right),(\mathbf{b})$ normalized mode area, $A_{m}$, (c) propagation length, $L_{p}$, and (d) figure of merit, $F o M$, of the present structure versus the operating frequency, $f$, for several values of $E_{F}$ when $w_{g}=200 \mathrm{~nm}, w_{r}=10 \mathrm{~nm}, h_{r}=30 \mathrm{~nm}, t_{S i}=5 \mathrm{~nm}$, and $t_{d}=5 \mathrm{~nm}$.

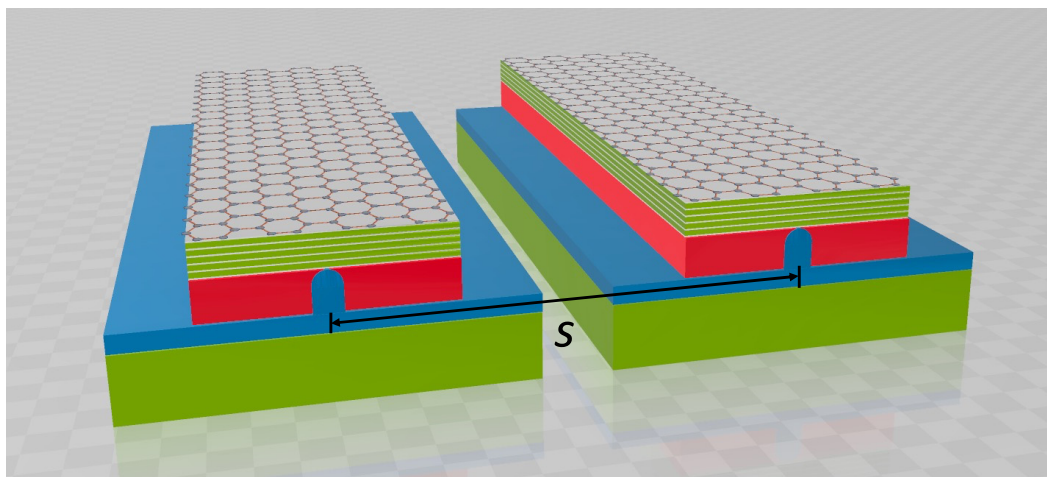

Figure 13. Schematic of a coupled waveguide consisting of two parallel MLGHPWs with a center-tocenter separation $s$.

According to the coupled mode theory (CMT) [58], energy exchange between the adjacent waveguides is due to the field coupling of evanescent tails of two normal modes. To evaluate the coupling strength, the coupling length of a lossless coupled waveguide system $L_{c}=\lambda /\left[2\left(n_{s}-n_{a}\right)\right]$ determines the length required to completely transfer power from one waveguide to another, where $n_{s}$ and $n_{a}$ are the $n_{e}$ of the symmetric and asymmetric modes, respectively. For a lossy waveguide system such as plasmonic waveguides, we adopted a more suitable criterion, the normalized coupling length $L_{c} / L_{\text {ave }}$ [59], which considers both the power attenuation and maximum power transfer to measure the crosstalk, where $L_{\text {ave }}$ is the average $L_{p}$ of the symmetric and asymmetric modes. The maximum transfer power $\rho_{\max }$ between waveguides is only a function of $L_{c} / L_{\text {ave }}$, and the adjacent waveguides can be considered as nearly isolated $\left(\rho_{\max }=0.33 \%\right)$ if $L_{c} / L_{\text {ave }}>10$ is reached. This is because the transfer power from one channel to the other is relatively weak at the distance of $L_{\text {ave }}$. At the frequency $f=30 \mathrm{THz}$ and the geometry parameters of $w_{g}=180 \mathrm{~nm}, w_{r}=20 \mathrm{~nm}$, $h_{r}=30 \mathrm{~nm}, t_{S i}=25 \mathrm{~nm}$, and $t_{d}=25 \mathrm{~nm}$, the results of $L_{c} / L_{\text {ave }}$ along with $\rho_{\max }$ versus $s$ at $\mu=1 \mathrm{~m}^{2} /$ V.s for several $E_{F} \mathrm{~s}$ are shown in Figure $14 \mathrm{a}$ and those at $E_{F}=0.4 \mathrm{eV}$ are shown in 
Figure $14 \mathrm{~b}$ for several $\mu \mathrm{s}$. The results show that decreasing $E_{F}$ or $\mu$ leads to weaker coupling strength, and the dependence of coupling strength on $E_{F}$ is stronger than that on $\mu$. We observe that the separations for negligible couplings between waveguides are $s=0.52,0.64$, and $0.72 \mu \mathrm{m}$ for $E_{F}=0.35,0.40$, and $0.45 \mathrm{eV}$, respectively (see Figure 14a), and are $s=0.57$, 0.61 , and $0.64 \mu \mathrm{m}$ for $\mu=0.5,0.75$, and $1 \mathrm{~m}^{2} /$ V.s, respectively (see Figure $14 \mathrm{~b}$ ). The results demonstrated that the coupling strength can be tuned by varying $E_{F}$ or $\mu$, respectively. For instance, $\rho_{\max }$ achieves about $20 \%$ at $E_{F}=0.45 \mathrm{eV}$ and $5 \%$ at $E_{F}=0.35 \mathrm{eV}$ for the condition $\mu=1 \mathrm{~m}^{2} /$ V.s and $s=0.4 \mu \mathrm{m}$. The small values of $s$ (about 0.5 to $0.7 \mu \mathrm{m}$ ) make the proposed waveguide capable of building high-density graphene-based photonic-integrated circuits operating in the mid-infrared range.

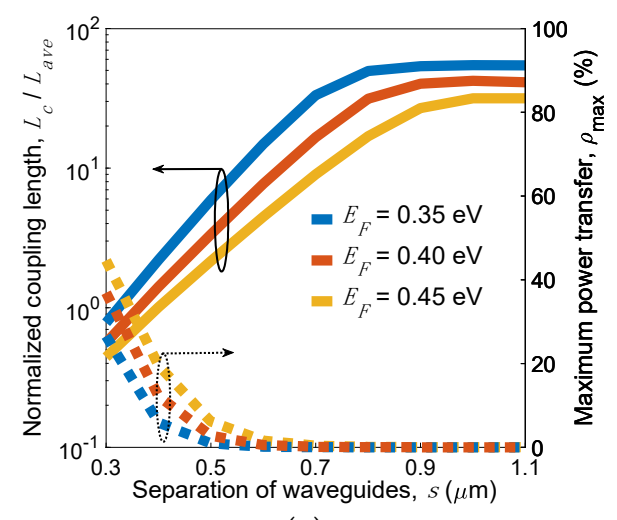

(a)

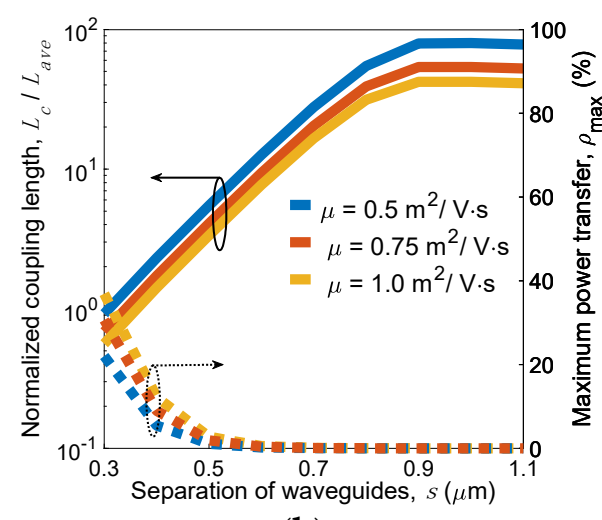

(b)

Figure 14. Normalized coupling length, $L_{c} / L_{\text {ave }}$ and maximum transfer power, $\rho_{\max }$ as functions of separation of waveguides, $s$ for several values of (a) $E_{F}$ at $\mu=1 \mathrm{~m}^{2} / \mathrm{V} \cdot \mathrm{s}$ and (b) $\mu$ at $E_{F}=0.4 \mathrm{eV}$ for $f=30 \mathrm{THz}$ and the geometry parameters $w_{g}=180 \mathrm{~nm}, w_{r}=20 \mathrm{~nm}, h_{r}=30 \mathrm{~nm}, t_{S i}=25 \mathrm{~nm}$, and $t_{d}=25 \mathrm{~nm}$.

\subsection{Comparison of Waveguiding Performance}

To demonstrate the superior waveguiding performance of the proposed design, we compared the reported results $[32,34,36,38,39]$ in Table 1 with our mode properties at the following parameters: $w_{g}=200 \mathrm{~nm}, w_{r}=10 \mathrm{~nm}, h_{r}=30 \mathrm{~nm}, t_{S i}=5 \mathrm{~nm}, t_{d}=5 \mathrm{~nm}, E_{F}=0.6 \mathrm{eV}$, and $N=10$. All the results in Table 1 were calculated at $f=30 \mathrm{THz}, \mu=1 \mathrm{~m}^{2} / \mathrm{V} \cdot \mathrm{s}$, and $E_{F}=0.6 \mathrm{eV}$, except the results in ref. [32] with $E_{F}=0.8 \mathrm{eV}$. If $E_{F}=0.8 \mathrm{eV}$ is decreased to $0.6 \mathrm{eV}, A_{m}$ and $L_{p}$ will be further reduced. Liu et al. [32] achieved an extremely small area, $A_{m}=8.0 \times 10^{-7}$, by adopting a symmetrical HPW. Teng et al. [36] proposed a GCNW dimer to cause the coupling of the fundamental modes between two GCNWs to improve the $A_{m}$ of the single GCNW [34] by one order of magnitude while keeping the same $L_{p}$. Liu et al. [38] extended their previous report [32] to add an extra graphene sheet between $\mathrm{Si}$ and $\mathrm{SiO}_{2}$ layers and roll it into a cylindrical waveguide. It can be inferred that the two-layer graphene structure effectively improves the $L_{p}$ but at the cost of a larger $A_{m}$. Last year, Teng et al. [39] proposed a GCNWLSNR structure composed of a single GCNW deposited on a Si nano-rib, which showed a performance of $L_{p}=3.5 \mu \mathrm{m}$ and $A_{m}=2.0 \times 10^{-6}$. In comparison with these published results $[32,34,36,38,39]$, the proposed structure achieves an unprecedented waveguiding performance of $L_{p}=19.4 \mu \mathrm{m}$ and $A_{m}=2.6 \times 10^{-6}$, thus obtaining an extremely high value of $F o M=10,612$. 
Table 1. Comparisons of the modal properties of $A_{m}, L_{p}$, and FoM.

\begin{tabular}{ccccc}
\hline Reference & $\boldsymbol{A}_{\boldsymbol{m}}$ & $\boldsymbol{L}_{\boldsymbol{p}}(\boldsymbol{\mu \mathrm { m } )}$ & $\boldsymbol{F o M}$ & $\boldsymbol{E}_{\boldsymbol{F}}(\mathbf{e V})$ \\
\hline$[32]$ & $8.0 \times 10^{-7}$ & 5 & 4951 & 0.8 \\
{$[34]$} & $9.0 \times 10^{-4}$ & 4 & 118 & 0.6 \\
{$[36]$} & $9.0 \times 10^{-5}$ & 4 & 374 & 0.6 \\
{$[38]$} & $1.0 \times 10^{-5}$ & 10 & 2803 & 0.6 \\
{$[39]$} & $2.0 \times 10^{-6}$ & 3.5 & 2193 & 0.6 \\
This work & $2.6 \times 10^{-6}$ & 19.4 & 10,612 & 0.6 \\
\hline
\end{tabular}

Currently, the obstacles for experimentally fabricating integrated graphene waveguides include three major points, despite the mature developments of both silicon photonics and graphene industries. (1) Excitation of extremely high- $k$ SPP modes supported by monolayer graphene; (2) high-efficiency coupling between the nanoscale mode sizes of high- $k$ SPP modes and submicron-scale dielectric waveguide modes or micron-scale optical fiber modes; (3) sufficient long propagation length beyond hundreds of micrometers or even millimeters. To relieve the first obstacle, employing multilayer graphene structures can efficiently control the field localizations and effective refractive index by varying the number of the graphene layers, thus overcoming the difficulty of exciting the high- $k$ SPP modes. Next, the coupling efficiency can be improved by designing perfectly adiabatic metallic gratings, reducing field scattering during the coupling process. The final one is a common limit, as the SPP modes supported by general noble metals operating in the near-IR and visible light bands. In addition to discovering new low-loss materials, designing a novel waveguide structure by combining multiple guiding mechanisms together has been the most effective solution to decrease the propagation losses.

\section{Conclusions}

This work reported a mid-IR waveguiding structure based on MLGMTs on a $\mathrm{Si}$ nano-rib waveguide structure covered by a porous $\mathrm{SiO}_{2}$ layer. By coupling the low-loss fundamental VPP mode of the MLGMTs, which is formed by coupling the SPP modes at individual graphene sheets and the dielectric mode of a Si nano-rib, the hybrid mode of the present design achieves an ultralong propagation length $L_{p}=19.4 \mu \mathrm{m}$ with $A_{m}=2.6 \times 10^{-6}$ at $E_{F}=0.6 \mathrm{eV}$ operating at $f=30 \mathrm{THz}$. Compared with the reported results, the $L_{p}$ of our structure is five times greater than those reported at a comparable $A_{m}$. Even for the looser $A_{m}$, previously reported $L_{p}$ values at $E_{F}=0.6 \mathrm{eV}$ and $f=30 \mathrm{THz}$ were still limited to below $10 \mu \mathrm{m}$. The MLGMTs provide a high-conductivity graphene structure that significantly increases $L_{p}$ with an increasing number of graphene layers. Therefore, the increased degree of $L_{p}$ is mainly restricted by the modern fabrication technique. In addition, the crosstalk between two adjacent waveguides demonstrates that the proposed structure is beneficial in realizing high-integration photonic devices operating in the mid-IR band. Our design is expected to pave the way for potential applications in building ultralow loss and compact and tunable mid-IR photonic devices and can be extended to other extraordinary $2 \mathrm{D}$ materials.

Author Contributions: All authors conceived the designed idea and discussed the results. R.-J.C. and C.-W.C. performed the numerical simulations. C.-C.H. wrote the manuscript and supervised the whole work. All authors have read and agreed to the published version of the manuscript.

Funding: This research was funded by the Ministry of Science and Technology (MOST) of Taiwan for financially supporting this research under grant number 110-2112-M-005-005.

Institutional Review Board Statement: Not applicable.

Informed Consent Statement: Not applicable.

Data Availability Statement: The data presented in this study are available on request from the corresponding author. 
Acknowledgments: The authors would like to thank Yen-Fu Lin in the Institute of Nanoscience, National Chung Hsing University, for the insight discussions of the fabrication processes of multilayer graphene metamaterials.

Conflicts of Interest: The authors declare no conflict of interest.

\section{References}

1. Barnes, W.L.; Dereux, A.; Ebbesen, T.W. Surface plasmon subwavelength optics. Nature 2003, 424, 824-830. [CrossRef]

2. Gramotnev, D.K.; Bozhevolnyi, S.I. Plasmonics beyond the diffraction limit. Nat. Photonics 2010, 4, 83-91. [CrossRef]

3. Han, Z.; Bozhevolnyi, S.I. Radiation guiding with surface plasmon polaritons. Rep. Prog. Phys. 2013, 76, 016402. [CrossRef]

4. Bian, Y.; Gong, Q. Highly confined guiding of low-loss plasmon waves in hybrid metal-dielectric slot waveguides. Nanotechnology 2014, 25, 345201. [CrossRef]

5. Bian, Y.; Ren, Q.; Kang, L.; Yue, T.; Werner, P.L.; Werner, D.H. Deep-subwavelength light transmission in hybrid nanowire-loaded silicon nano-rib waveguides. Photonics Res. 2018, 6, 37-45. [CrossRef]

6. Stanley, R. Plasmonics in the mid-infrared. Nat. Photonics 2012, 6, 409-411. [CrossRef]

7. Zhong, Y.; Malagari, S.D.; Hamilton, T.; Wasserman, D.M. Review of mid-infrared plasmonic materials. J. Nanophotonics 2015, 9, 093791. [CrossRef]

8. Grigorenko, A.N.; Polini, M.; Novoselov, K.S. Graphene plasmonics. Nat. Photonics 2012, 6, 749-758. [CrossRef]

9. Low, T.; Avouris, P. Graphene plasmonic for terahertz to mid-infrared applications. ACS Nano 2014, 8, 1086-1101. [CrossRef] [PubMed]

10. Politano, P.; Chiarello, G. Plasmon modes in graphene: Status and prospect. Nanoscale 2014, 6, 10927-10940. [CrossRef] [PubMed]

11. Gon, P.A.D.; Peres, N.M.R. An Introduction to Graphene Plasmonics; World Scientific: Singapore, 2016.

12. Li, Y.; Tantiwanichapan, K.; Swan, A.K.; Paiella, R. Graphene plasmonic devices for terahertz optoelectronics. Nanophotonics 2020, 9, 1901-1920. [CrossRef]

13. Bao, Q.; Zhang, H.; Wang, B.; Ni, Z.; Haley, C.; Lim, Y.X.; Wang, Y.; Tang, D.Y.; Loh, K.P. Broadband graphene polarizer. Nat. Commun. 2011, 5, 411-415. [CrossRef]

14. Pei, C.; Yang, L.; Wang, G.; Wang, Y.; Jiang, X.; Hao, Y.; Li, Y.; Yang, J. Broadband graphene/glass hybrid waveguide polarizer. IEEE Photonics Technol. Lett. 2015, 27, 927-930. [CrossRef]

15. He, X.; Liu, J. Flexible and broadband graphene polarizer based on surface silicon-core microfiber. Opt. Mater. Express 2017, 7, 1398-1405. [CrossRef]

16. Kim, J.T.; Choi, H. Polarization control in graphene-based polymer waveguide polarizer. Laser Photonics Rev. 2018, 12, 1800142. [CrossRef]

17. Liu, M.; Yin, X.; Ulin-Avila, E.; Geng, B.; Zentgraf, T.; Ju, L.; Wang, F.; Zhang, X. A graphene-based broadband optical modulator. Nature 2011, 474, 64-67. [CrossRef]

18. Youngblood, N.; Anugrah, Y.; Ma, R.; Koester, S.K.; Li, M. Multifunctional graphene optical modulator and photodetector integrated on silicon waveguides. Nano Lett. 2014, 14, 2741-2746. [CrossRef]

19. Ansell, D.; Radko, I.P.; Han, Z.; Rodriguez, F.J.; Bozhevolnyi, S.I.; Grigorenko, A.N. Hybrid graphene plasmonic waveguide modulators. Nat. Commun. 2015, 6, 8846. [CrossRef]

20. Hao, R.; Jiao, J.; Peng, X.; Zhen, Z.; Dagarbek, R.; Zou, Y.; Li, E. Experimental demonstration of a graphene-based hybrid plasmonic modulator. Opt. Lett. 2019, 44, 2586-2589. [CrossRef]

21. Ding, Y.; Guan, X.; Zhu, X.; Hu, H.; Bozhevolnyi, S.I.; Oxenløwe, L.K.; Jin, K.J.; Mortensen, N.A.; Xiao, S. Efficient electro-optic modulation in low-loss graphene-plasmonic slot waveguides. Nanoscale 2017, 9, 15576-15581. [CrossRef]

22. Yuana, W.; Shi, G. Graphene-based gas sensors. J. Mater. Chem. A 2013, 1, 10078-10091. [CrossRef]

23. Peters, A.; Turvey, S.; Horsfall, A.B. High-temperature Hall effect sensor based on epitaxial graphene on high-purity semiinsulating 4H-SiC. IEEE Trans. Electron Dev. 2019, 66, 3134-3138.

24. Choi, J.H.; Lee, J.; Byeon, M.; Hong, T.E.; Park, H.; Lee, C.Y. Graphene-based gas sensors with high sensitivity and minimal sensor-to-sensor variation. ACS Appl. Nano Mater. 2020, 3, 2257-2265. [CrossRef]

25. Ghorbanzadeh, M.; Darbari, S.; Moravvej-Farshia, M.K. Graphene-based plasmonic force switch. Appl. Phys. Lett. 2016, 108, 111105. [CrossRef]

26. Dolleman, R.J.; Belardinelli, P.; Houri, S.; van der Zant, H.S.J.; Alijani, F.; Steeneken, P.G. High-frequency stochastic switching of graphene resonators near room temperature. Nano Lett. 2019, 19, 1282-1288. [CrossRef] [PubMed]

27. Cox, J.D.; de Abajo, F.J.G. Single-plasmon thermo-optical switching in graphene. Nano Lett. 2019, 19, 3743-3750. [CrossRef] [PubMed]

28. Ono, M.; Hata, M.; Tsunekawa, M.; Nozaki, K.; Sumikura, H.; Chiba, H.; Notomi, M. Ultrafast and energy-efficient all-optical switching with graphene-loaded deep-subwavelength plasmonic waveguides. Nat. Photonics 2020, 14, 37-43. [CrossRef]

29. Ye, L.; Sui, K.; Feng, H. High-efficiency couplers for graphene surface plasmon polaritons in the mid-infrared region. Opt. Lett. 2020, 45, 264-267. [CrossRef]

30. Sun, Y.; Bian, Y.; Zhao, X.; Zheng, Z.; Liu, J.; Liu, J. Low-loss graphene plasmonic waveguide based on a high-index dielectric wedge for tight optical confinement. In Proceedings of the OSA Technical Digest (Optical Society of America, 2013), online, 9-14 June 2013; p. JTu4A.33. 
31. Xu, W.; Zhu, Z.H.; Liu, K.; Zhang, J.F.; Yuan, X.D.; Lu, Q.S.; Qin, S.Q. Dielectric loaded graphene plasmon waveguide. Opt. Express 2015, 23, 5147-5153. [CrossRef]

32. Liu, J.P.; Zhai, X.; Wang, L.L.; Li, H.J.; Xie, F.; Xia, S.X.; Shang, X.J.; Luo, X. Graphene-based long-range SPP hybrid waveguide with ultra-long propagation length in mid-infrared range. Opt. Express 2016, 24, 5376-5386. [CrossRef]

33. Bahadori-Haghighi, S.; Ghayour, R.; Sheikhi, M.H. Design and analysis of low loss plasmonic waveguide and directional coupler based on pattern-free suspended graphene sheets. Carbon 2018, 129, 653-660. [CrossRef]

34. Gao, Y.; Ren, G.; Zhu, B.; Wang, J.; Jian, S. Single-mode graphene-coated nanowire plasmonic waveguide. Opt. Lett. 2014, 39, 5909-5912. [CrossRef]

35. Gao, Y.; Ren, G.; Zhu, B.; Liu, H.; Lian, Y.; Jian, S. Analytical model for plasmon modes in graphene-coated nanowire. Opt. Express 2014, 22, 24322-24331. [CrossRef]

36. Teng, D.; Wang, K.; Li, Z.; Zhao, Y. Graphene-coated nanowire dimers for deep subwavelength waveguiding in mid-infrared range. Opt. Express 2019, 27, 12458-12469. [CrossRef] [PubMed]

37. Liu, J.P.; Zhai, X.; Wang, L.L.; Li, H.J.; Xie, F.; Lin, Q.; Xia, S.X. Analysis of mid-infrared surface plasmon modes in a graphene-based cylindrical hybrid waveguide. Plasmonics 2016, 11, 703-711. [CrossRef]

38. Liu, J.P.; Zhai, X.; Xie, F.; Wang, L.L.; Xia, S.X.; Li, H.J.; Luo, X.; Shang, X.J. Analytical model of mid-infrared surface plasmon modes in a cylindrical long-range waveguide with double-layer graphene. J. Lightwave Technol. 2017, 35, 1971-1979. [CrossRef]

39. Teng, D.; Wang, K.; Huan, Q.; Chen, W.; Li, Z. High-performance light transmission based on graphene plasmonic waveguides. J. Mater. Chem. C 2020, 8, 6832-6838. [CrossRef]

40. Oulton, R.F.; Sorger, V.J.; Genov, D.; Pile, D.; Zhang, X. A hybrid plasmonic waveguide for subwavelength confinement and long-range propagation. Nat. Photonics 2008, 2, 496-500. [CrossRef]

41. Chen, B.; Meng, C.; Yang, Z.; Li, W.; Lin, S.; Gu, T.; Guo, X.; Wang, D.; Yu, S.; Wong, C.W. Graphene coated ZnO nanowire optical waveguides. Opt. Express 2014, 22, 24276-24285. [CrossRef] [PubMed]

42. Cao, T.; Tian, L.; Liang, H.; Qin, K.R. Reconfigurable, graphene-coated, chalcogenide nanowires with a sub-10-nm enantioselective sorting capability. Microsyst. Nanoeng. 2018, 4, 7. [CrossRef]

43. Iorsh, I.V.; Mukhin, I.S.; Shadrivov, I.V.; Belov, P.A.; Kivshar, Y.S. Hyperbolic metamaterials based on multilayer graphene structures. Phys. Rev. B 2013, 87, 075416. [CrossRef]

44. Othman, M.A.K.; Guclu, C.; Capolino, F. Graphene-based tunable hyperbolic metamaterials and enhanced near-field absorption. Opt. Express 2013, 21, 7614-7632. [CrossRef] [PubMed]

45. Smirnova, D.A.; Iorsh, I.V.; Shadrivov, I.V.; Kivshar, Y.S. Multilayer graphene waveguides. JETH Lett. 2014, 99, 456. [CrossRef]

46. Schubert, E.F.; Kim, J.K.; Xi, J.Q. Low-refractive-index materials: A new class of optical thin-film materials. Phys. Status Solidi B 2007, 244, 3002-3008. [CrossRef]

47. Lin, J.Y.; Liu, W.; Smart, J.A. Optical thin-film materials with low refractive index for broadband elimination of Fresnel reflection. Nat. Photonics 2007, 1, 176-179.

48. Yan, H.; Li, X.; Chandra, B.; Tulevski, G.; Wu, Y.; Freitag, M.; Zhu, W.; Avouris, P.; Xia, F. Tunable infrared plasmonic devices using graphene/insulator stacks. Nat. Nanotechnol. 2012, 7, 330-334. [CrossRef]

49. Chang, Y.C.; Liu, C.H.; Liu, C.H.; Zhang, S.; Marder, S.R.; Narimanov, E.E.; Zhong, Z.; Norris, T.B. Realization of mid-infrared graphene hyperbolic metamaterials. Nat. Commun. 2015, 7, 10568. [CrossRef] [PubMed]

50. Baitimirova, M.; Viter, R.; Andzane, J.; Lee, A.; Voiry, D.; Iatsunskyi, I.; Coy, E.; Mikoliunaite, L.; Tumenas, S.; Załęski, K.; et al Tuning of structural and optical properties of graphene/ZnO nanolaminates. J. Phys. Chem. C 2016, 120, 23716-23725. [CrossRef]

51. Yang, Y.; Lin, H.; Zhang, B.Y.; Zhang, Y.; Zheng, X.; Yu, A.; Hong, M.; Jia, B. Graphene-based multilayered metamaterials with phototunable architecture for on-chip photonic devices. ACS Photonics 2019, 6, 1033-1040. [CrossRef]

52. Lin, H.; Sturmberg, B.C.P.; Lin, K.; Yang, Y.; Zheng, X.; Chong, T.K.; Martijn de Sterke, C.; Jia, B. A 90-nm-thick graphene metamaterial for strong and extremely broadband absorption of unpolarized light. Nat. Photonics 2019, 13, 270-276. [CrossRef]

53. Lee, C.C.; Suzuki, S.; Xie, W.; Schibli, T.R. Broadband graphene electro-optic modulators with sub-wavelength thickness. Opt. Express 2012, 20, 5264-5269. [CrossRef] [PubMed]

54. Fan, M.; Yang, H.; Zheng, P.; Hu, G.; Yun, B.; Cui, Y. Multilayer graphene electro-absorption optical modulator based on double-stripe silicon nitride waveguide. Opt. Express 2017, 25, 21619-21629. [CrossRef] [PubMed]

55. Sun, D.; Divin, C.; Berger, C.; De Heer, W.A.; First, P.N.; Norris, T.B. Spectroscopic measurement of interlayer screening in multilayer epitaxial graphene. Phys. Rev. Lett. 2010, 104, 136802. [CrossRef] [PubMed]

56. Bass, M.; DeCusatis, C.; Enoch, J.; Lakshminarayanan, V.; Li, G.; MacDonald, C.; Mahajan, V.; StrylandBass, E.V. Handbook of Optics, Third Edition Volume IV: Optical Properties of Materials, Nonlinear Optics, Quantum Optics; McGraw-Hill: New York, NY, USA, 2009.

57. Falkovsky, L.A.; Pershoguba, S.S. Optical far-infrared properties of a graphene monolayer and multilayer. Phys. Rev. B 2007, 76,153410 . [CrossRef]

58. Huang, W.P. Coupled-mode theory for optical waveguides: An overview. J. Opt. Soc. Am. A 1994, 11, 963-983. [CrossRef]

59. Veronis, G.; Fan, S.H. Crosstalk between three-dimensional plasmonic slot waveguides. Opt. Express 2008, 16, 2129-2140. [CrossRef] 